\title{
An update on clinical utility of rilpivirine in the management of HIV infection in treatment-naïve patients
}

This article was published in the following Dove Press journal:

HIVIAIDS - Research and Palliative Care

12 September 2013

Number of times this article has been viewed

\author{
Opass Putcharoen ${ }^{1-3}$ \\ Stephen J Kerr ${ }^{4}$ \\ Kiat Ruxrungtham ${ }^{2,4,5}$ \\ 'Division of Infectious Diseases, \\ Department of Medicine, \\ Chulalongkorn University, ${ }^{2}$ Faculty \\ of Medicine, King Chulalongkorn \\ Memorial Hospital, Thai Red Cross \\ Society, Chulalongkorn University, \\ ${ }^{3}$ Center of Excellence for Infectious \\ Diseases, King Chulalongkorn \\ Memorial Hospital, Thai Red \\ Cross Society, ${ }^{4}$ HIV-NAT, Thai Red \\ Cross, AIDS Research Center, \\ ${ }^{5}$ Division of Allergy and Clinical \\ Immunology, Department of Medicine, \\ Chulalongkorn University, Bangkok, \\ Thailand
}

\begin{abstract}
Non-nucleoside analog reverse transcriptase inhibitors (NNRTIs) are an important component of combination antiretroviral regimens. Amongst the NNRTIs, efavirenz is commonly recommended for initial regimens in treatment-naïve HIV patients, but its use in some settings is limited by adverse effects, particularly those affecting the central nervous system and lipid metabolism. Rilpivirine is a new second-generation NNRTI that is recommended as an alternative to efavirenz in treatment-naïve HIV patients. Evidence of the clinical efficacy of rilpivirine versus efavirenz, in combination with two nucleoside or nucleotide analog reverse transcriptase inhibitors in treatment-naïve patients, is derived from the THRIVE and ECHO studies. These studies demonstrated that rilpivirine $25 \mathrm{mg}$ once daily was potent and noninferior to efavirenz $600 \mathrm{mg}$ once daily using an intention-to-treat time-to-loss-of-virologicresponse (ITT-TLOVR) endpoint. Although virologic failure was higher in subjects treated with rilpivirine, study discontinuations due to adverse effects were more common in subjects treated with efavirenz. In addition, the virologic response to rilpivirine was suboptimal in patients with a baseline viral load $>100,000$ copies $/ \mathrm{mL}$. The overall incidence of adverse events and grade 2-4 adverse events was lower in the rilpivirine than in the efavirenz groups. Patients with rilpivirine failure were more likely to have resistance mutations that confer crossresistance to other NNRTIs, including etravirine. Rilpivirine is currently available as a fixed-dose combination that allows for once-daily administration as a single pill, and is approved for use in treatment-naïve patients. This drug is contraindicated when co-administered with rifamycins or proton-pump inhibitors.
\end{abstract}

Keywords: rilpivirine, HIV infection, treatment-naïve

\section{Introduction}

Advances in antiretroviral therapy (ART) have ultimately improved the survival of HIV-infected patients, but the viral latency of HIV is a barrier for HIV eradication or cure. ${ }^{1-3}$ Currently used antiretroviral regimens can control HIV replication and maintain maximal viral suppression if patients adhere to this chronic and life-long treatment. However, various factors can compromise HIV treatment success. First, after a long duration of exposure to ART, some patients experience long-term adverse effects from antiretroviral agents. ${ }^{4,5}$ Second, adherence to prolonged ART may decrease over time. ${ }^{6}$ Some ART regimens contain a higher pill burden that may further compromise adherence, ${ }^{7,8}$ but most of the antiretroviral agents currently recommended for treatmentnaïve patients are potent, durable, easy to administer, and have a low incidence of toxicity. ${ }^{9}$ New agents should be safe for use in particular settings such as pregnancy or childbearing women. In this review, we aim to update the evidence base about a
Correspondence: Kiat Ruxrungtham Department of Medicine, Faculty of Medicine, Chulalongkorn University and HIV-NAT, Thai Red Cross, AIDS Research Center, Bangkok 10330, Thailand Email kiat.r@chula.ac.th 
novel non-nucleoside analog reverse transcriptase inhibitor (NNRTI), rilpivirine, in treatment-naïve HIV patients, with data from relevant clinical trials.

\section{The discovery of the second- generation NNRTI}

Antiretroviral regimens usually consist of two nucleoside analog reverse transcriptase inhibitors (NRTI) plus a third agent. The recommended drug classes for selection of the third agent are protease inhibitors (PIs), NNRTIs, and integrase strand transfer inhibitors. ${ }^{9}, 10$ Although there are numerous choices available within these classes, only some agents are widely used and recommended in treatment-naïve patients as preferred third agents. More specifically, atazanavir boosted with ritonavir, darunavir boosted with ritonavir, efavirenz, and raltegravir are recommended in treatment-naïve patients as preferred third agents in the highly active ART (HAART) combination for developed countries. Amongst the NNRTIs, efavirenz and nevirapine have high potency and tolerability, ${ }^{11}$ but efavirenz is recommended as the preferred third agent in the NNRTI class, because of demonstrated high efficacy in many clinical trials, lower rates of toxicity, and pharmacokinetic properties that allow for once-daily dosing. ${ }^{9}$ Efavirenz has been assigned as a gold standard or comparator for study of the efficacy of new antiretroviral agents. However, its use can be limited by central nervous system (CNS) adverse effects, ${ }^{12}$ cutaneous eruptions, and alterations in lipid metabolism. ${ }^{5}$ In addition, efavirenz is teratogenic in animals, and might be associated with congenital anomalies in humans. ${ }^{13}$ Other preferred 'third agents' for treatment-naïve patients also have limitations. PIs such as boosted atazanavir or boosted darunavir may cause dyslipidemia. ${ }^{14,15}$ Raltegravir is potent but still requires twice-daily dosing. ${ }^{16}$ Because of the limitations of current preferred agents in some clinical settings, there has been a search for effective new agents with improved toxicity profiles. Etravirine is the first of the second-generation NNRTIs that has showed efficacy in controlling HIV replication in treatment-experienced patients, in combination with other active agents. ${ }^{17-19}$ In this review, we provide an update on the clinical use of rilpivirine, the second of the second-generation NNRTIs, a recommended alternative third agent for treatment-naïve patients.

\section{The pharmacological properties of rilpivirine}

Rilpivirine acts at the hydrophobic position near the NNRTIbinding site, causing inactivation of the reverse transcriptase enzyme, thus terminating DNA synthesis of the HIV virus. ${ }^{20,21}$ Rilpivirine shares some chemical similarities with etravirine and hence these two agents have potential cross-resistance. ${ }^{22}$ Etravirine has a higher genetic barrier to resistance and may be more suitable for use in treatment-experienced patients, while rilpivirine has a long terminal half-life, allowing for once-daily dosing, and is therefore a suitable choice for treatment-naïve patients. ${ }^{23}$ Rilpivirine $25 \mathrm{mg}$ once daily is the only dose licensed by the US Food and Drug Administration (FDA), due to suspected QT interval problems with higher doses in Phase I and II studies. ${ }^{24}$

\section{Evidence of rilpivirine efficacy from clinical trials}

In dose-finding studies, $25 \mathrm{mg}$ of rilpivirine had viral suppression comparable to that of efavirenz through 96 weeks. ${ }^{24,25}$ Efficacy of rilpivirine was subsequently determined in the THRIVE (TMC278 against HIV, in a once daily RegImen Versus Efavirenz) and ECHO (Early Capture HIV Cohort Study) studies. ${ }^{26,27}$ These two similar Phase III, multinational, double-blinded, randomized, placebocontrolled, non-inferiority studies compared the efficacy of rilpivirine versus efavirenz in combination with a 2-NRTI backbone. The primary endpoint of both studies was an 'intention-to-treat time to loss-of-virologic response' (ITTTLOVR) algorithm, and the secondary endpoints were rates of adverse effects, changes in HIV-1 viral load, CD4 cell counts, lipid parameters from baseline and patterns of drug resistance-associated mutations. The choice of backbone regimen in THRIVE was at the investigators' discretion, but, in ECHO, the backbone was tenofovir/emtricitabine. The response rate in both trials was stratified by backbone regimens and baseline viral load $(<100,000$ copies $/ \mathrm{mL}$, 100,001-500,000 copies $/ \mathrm{mL}$, and $>500,000$ copies $/ \mathrm{mL}$ ). A total of 1,368 subjects were enrolled in both studies, and 1,062 subjects were followed to week $48 ; 80 \%$ of subjects were male, with a balanced gender ratio in both studies, and approximately $50 \%$ of subjects had a baseline HIV-1 viral load greater than 100,000 copies $/ \mathrm{mL}$. The backbone regimens in THRIVE were tenofovir/emtricitabine, zidovudine/ lamivudine, and abacavir/lamivudine. The mean HIV-1 viral load was $5 \log _{10}$ copies $/ \mathrm{mL}$, and median CD4 cell count was 250 cells $/ \mathrm{mm}^{3,28}$

In the THRIVE study, the observed response rates were $86 \%$ for rilpivirine- and $82 \%$ for efavirenz-treated subjects. The ECHO study also showed similar results, with response rates of $83 \%$ and $83 \%$ for rilpivirine and efavirenz, respectively. In pooled analysis, the response 
rate for rilpivirine was $78 \%$ and for efavirenz was $78 \% .^{29}$ Rilpivirine was potent and non-inferior to efavirenz in both studies. The ITT-TLOVR primary outcome included subjects who had virologic failure (rebound/never suppressed) or discontinued study drug for any reason. The rate of efavirenz discontinuation was higher than that observed with rilpivirine in both studies, but the rate of viral failure due to rebound or failure to ever suppress was higher in the rilpivirine versus the efavirenz group ( $9 \%$ and $5 \%$, respectively). In summary, rilpivirine $25 \mathrm{mg}$ once daily and efavirenz $600 \mathrm{mg}$ once daily had comparable responses at week 96. Although more virologic failures were observed in the rilpivirine group, the tolerability was better than that observed in efavirenz-treated patients. The majority of virologic failures in the rilpivirine group occurred in the first 48 weeks.

In secondary endpoint analyses of THRIVE and ECHO, mean CD4 cell count increases from baseline were comparable in both treatment arms. A pooled analysis showed that response rates were worse in patients with lower baseline CD4 cell counts, and this effect was more prominent in the rilpivirine group. ${ }^{29}$ In THRIVE, $91 \%$ of patients with a baseline viral load of $<100,000$ copies/mL, $80 \%$ of those with a baseline viral load of 100,000-500,000 copies $/ \mathrm{mL}$, and $77 \%$ of those with a baseline viral load $>500,000$ copies $/ \mathrm{mL}$ responded in the rilpivirine group, and the proportion of responders in the efavirenz group in each viral load stratum was $84 \%, 82 \%$, and $69 \%$, respectively. In $\mathrm{ECHO}$, the corresponding numbers of responders in the rilpivirine group were $90 \%, 79 \%$, and $62 \%$ for baseline viral loads of $<100,000$ copies $/ \mathrm{mL}, 100,000-500,000 \mathrm{cop}-$ ies $/ \mathrm{mL}$, and $>500,000$ copies $/ \mathrm{mL}$, respectively, and $83 \%$, $83 \%$, and $81 \%$, respectively, for each viral load stratum in subjects treated with efavirenz (Figure 1). Thus, despite comparable CD4 responses between the two arms, virologic response was reduced in the rilpivirine group when baseline viral load was $>100,000$ copies $/ \mathrm{mL}$. Discontinuation rates due to adverse events were higher in the efavirenz than in the rilpivirine group; grade 2-4 adverse events were more common in the efavirenz than in the rilpivirine group. In THRIVE, the incidence of mild-to-moderate adverse effects was identical between the rilpivirine and efavirenz groups $(92 \%)$, but incidence of grade 2-4 adverse effects associated with treatment was lower in the rilpivirine than in the efavirenz group. Among common treatment-related (grade 2 or higher) adverse events, the incidence of rash in the rilpivirine group was significantly lower, and the incidence of neuropsychiatric adverse events was higher in the efavirenz group. These findings were similar in ECHO, with a higher incidence of grade 2-4 adverse effects associated with treatment in the efavirenz group. Among common treatment-related (grade 2 or higher) adverse events, the incidence of rash in the rilpivirine and efavirenz groups was $2 \%$ and $8 \%$, respectively $(P<0.001)$. Mean changes in lipid parameters, total cholesterol, low-density lipoprotein cholesterol and triglycerides from baseline to week 48 after initiation of treatment were significantly lower in the rilpivirine group in THRIVE. In ECHO, the rate of discontinuation due to adverse events was six patients $(2 \%)$ in the rilpivirine group and 25 patients $(7 \%)$ in the efavirenz group. More patients in the efavirenz group had grade $2-4$ adverse events than in the rilpivirine group. The change in triglycerides was not significantly different between the two treatment arms.

\section{Patterns of rilpivirine-associated mutations and response to treatment in patients with primary NNRTI resistance}

A resistance analysis from THRIVE and ECHO demonstrated that the most common NNRTI-resistant mutation that emerged when subjects failed rilpivirine was E138K (77\%), and $\mathrm{K} 103 \mathrm{~N}$ (57\%) in subjects who failed efavirenz. A unique pattern of NRTI-associated mutations that emerged when failing rilpivirine was M184I or M184V/I mixtures. This pattern is also found in etravirine resistance, the M184I that co-emerges with E138K facilitates the replication capacity of resistant viruses. ${ }^{30}$ In addition, E138K and M184V/I that emerged in patients who have virologic failure to rilpivirine may confer resistance to other NNRTIs such as efavirenz, nevirapine, and etravirine. ${ }^{31,32}$ In patients who failed efavirenz with only the K103N mutation, viruses still maintained susceptibility to etravirine. ${ }^{17}$

Currently, there are concerns over transmitted (primary) HIV drug resistance and virologic response after initiation of ART. The prevalence of primary drug resistance in western countries is approximately $10 \%$ and may be higher in particular areas. ${ }^{33}$ Many studies have shown a reduced virologic response associated with transmitted drug-resistant viruses. ${ }^{34}$ Response to rilpivirine at week 48 in the THRIVE and ECHO studies was not affected by pre-existing NNRTI mutations, due to low prevalence of rilpivirine resistanceassociated mutations. ${ }^{35}$ This suggests rilpivirine may have a role in treatment-naïve patients in settings with a high or increasing prevalence of primary resistance from firstgeneration NNRTI-associated mutations. 

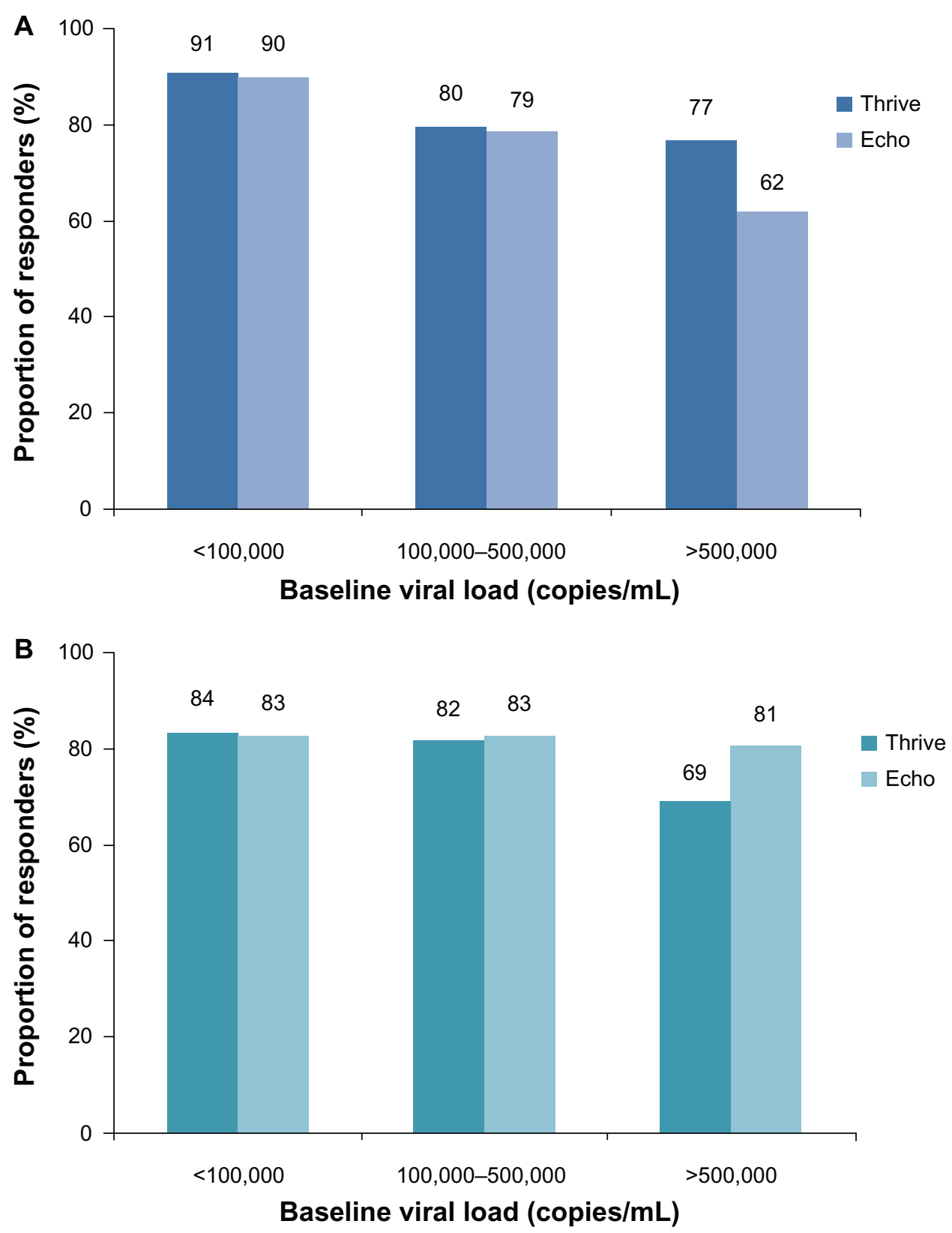

Figure I Proportion of responders to rilpivirine $(\mathbf{A})$ and efavirenz $(\mathbf{B})$ in the THRIVE and ECHO studies. Response rate in subjects in the rilpivirine group was reduced when baseline viral load was $>100,000$ copies $/ \mathrm{mL}^{26,27}$

Abbreviations: THRIVE, TMC278 against HIV, in a once daily Reglmen Versus Efavirenz; ECHO, Early Capture HIV Cohort Study.

\section{Patterns of response and use} of rilpivirine among different populations

No differences in response rates for subjects in either group were noted in subjects stratified by backbone regimen, gender, race, and HIV subtypes. ${ }^{36}$ However, it appeared that Asian subjects and those infected with HIV-1 CRF01_AE had higher response rates in both treatment arms. ${ }^{28} \mathrm{~A}$ subsequent pharmacodynamic study found that rilpivirine exposure was higher in female and Asian populations. ${ }^{36}$ Patients with hepatitis co-infection in both treatment arms had a higher rate of hepatic adverse events. $^{37}$

Although efavirenz causes fetal anomalies in animals and is classified as a US FDA pharmaceutical pregnancy category D drug, rilpivirine has not demonstrated any increased teratogenic risk in animal fetuses at doses 15 and 70 times higher than those recommended in humans. Currently, rilpivirine is classified in pregnancy category B. Rilpivirine might therefore be an alternative option for pregnant women. Nevertheless, recent evidence has confirmed that efavirenz is safe in pregnant women and has 
been endorsed in the most recent British HIV Association (BHIVA) and World Health Organization (WHO) guidelines for women after the first trimester. ${ }^{38,39}$ Rilpivirine may be preferable for women taking contraceptives, due to a lack of significant drug interactions with norethindrone and ethinyl estradiol.

\section{Switching study of rilpivirine}

In the SPIRIT (Switching boosted PI to Rilpivirine Incombination with Truvada as a single tablet regimen) trial, improvement in lipid parameters was demonstrated in 476 subjects, 24 weeks after switching to a rilpivirinebased regimen. Most patients in this study had received a boosted-PI regimen for at least 6 months, with viral load $<50$ copies/mL before switching. Patients were randomized to receive tenofovir/emtricitabine/rilpivirine (TDF/FTC/RPV) or ritonavir-boosted PI plus two NRTIs for 24 weeks, then patients randomized to the PI-based arm were switched to TDF/FTC/RPV. The primary endpoint of this study was virologic suppression at week 24 after switching, and secondary endpoints were changes in CD4 cell count, safety, and fasting lipid parameters from baseline before switching. After switching, the rate of virologic suppression between the two arms was comparable. In addition, subjects who switched to TDF/FTC/RPV had favorable changes in lipid parameters, particularly in triglyceride levels (Figure 2). ${ }^{40}$

Neuropsychiatric adverse effects were less common in subjects treated with rilpivirine than in those treated with efavirenz in the THRIVE and ECHO studies. Thus, rilpivirine may be an alternative choice for patients with intolerable CNS adverse effects from efavirenz. However, there is a concern over the drug interaction between efavirenz and rilpivirine: a pharmacokinetic study demonstrated that efavirenz decreases the minimum rilpivirine concentration by $25 \%$. This interaction might reduce efficacy when switching from efavirenz to rilpivirine. However, virologic suppression at 12 weeks was maintained in all of 49 subjects who were stable on an efavirenz-based regimen, then switched to rilpivirine. ${ }^{41}$ Follow-up data at week 48 will provide additional information on the clinical significance of this interaction.

\section{Summary: the clinical use of rilpivirine for treatment-naïve patients - data from relevant clinical studies}

In THRIVE and ECHO, rilpivirine was non-inferior to efavirenz in treatment-naïve patients. However, the primary composite endpoint, combining either virologic failure or treatment discontinuation mandates that results should be interpreted with caution. Although the rate of virologic failure was higher in the rilpivirine group, overall efficacy was balanced by higher discontinuation rates in the efavirenz group. Based on resistance results from these studies, rilpivirine might be a preferred agent in patients with transmitted NNRTI resistance. In addition, the virologic efficacy of rilpivirine was reduced in those with baseline HIV-1 viral loads $>100,000$ copies $/ \mathrm{mL}$. These facts must be considered when starting ART, since the ultimate goal of HIV treatment is maximal viral suppression. Due to the higher rate of virologic failures in subjects

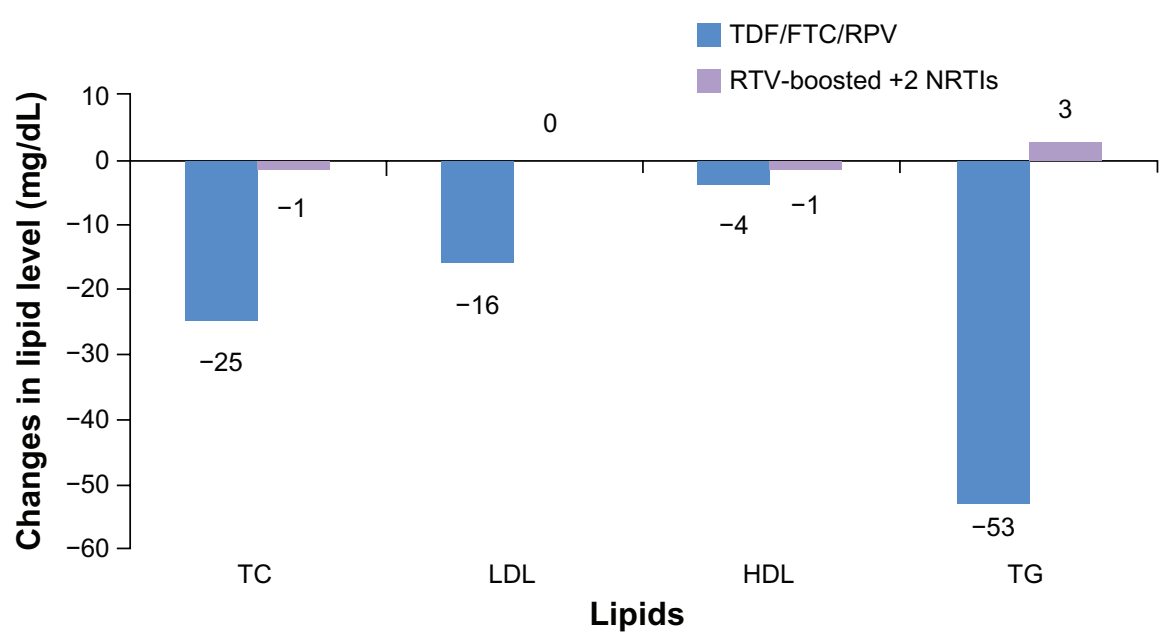

Figure 2 Lipid changes $(\mathrm{mg} / \mathrm{dL})$ in TDF/FTC/RPV versus continued ritonavir-boosted plus two NRTls at week 24 after switching.

Abbreviations: HDL, high-density lipoprotein; LDL, low-density lipoprotein; NRTIs, nucleoside analog reverse transcriptase inhibitors; TC, total cholesterol; TDF/FTC/ RPV, tenofovir/emtricitabine/rilpivirine; TG, triglyceride; RTV, ritronavir. 
taking rilpivirine than in those taking efavirenz, current US Department of Health and Human Services (DHHS) ART treatment guidelines for adults and adolescents recommend rilpivirine as an alternative initial regimen for treatment-naïve patients. ${ }^{10}$ The overall adverse effect rates and incidence of grade 2-4 events in the THRIVE and $\mathrm{ECHO}$ studies were lower in subjects taking rilpivirine than in those taking efavirenz. Adverse events leading to discontinuation of treatment were rash and depression, and these were higher in the efavirenz group; neurological adverse events such as dizziness and abnormal dreams were less common in subjects randomized to rilpivirine. In addition, mean change in lipid levels was lower in the rilpivirine group. This minimal effect on lipid metabolism of rilpivirine makes this agent suitable for patients with cardiovascular risk. Rilpivirine may be useful in patients who experience adverse effects such as hyperlipidemia or neuropsychiatric symptoms.

Since the most common rilpivirine-associated resistance mutations may have cross-resistance to other NNRTIs, including etravirine, patients failing rilpivirine would probably have fewer treatment options than patients who failed efavirenz. ${ }^{42}$

\section{Use of rilpivirine in a single-tablet regimen}

Use of once-daily ART is one strategy to improve adherence in HIV patients. Tenofovir/emtricitabine/efavirenz (TDF/FTC/EFV) is a well-tolerated option for single-tablet regimens (STRs). The STaR study aimed to compare two STRs: TDF/FTC/EFV versus TDF/FTC/RPV in treatmentnaïve patients for 96 weeks. The primary endpoint was the proportion of subjects with HIV-1 RNA $<50$ copies/mL at week 48 determined by the FDA snapshot algorithm (12\% pre-specified non-inferiority margin). A total of 784 subjects were enrolled and randomized. Baseline characteristics were well balanced in both treatment arms, with a baseline mean CD4 count of 390 cells $/ \mathrm{mm}^{3}$ and HIV-1 RNA of $4.8 \log _{10}$ copies/mL. The analysis showed that TDF/FTC/RPV was non-inferior to TDF/FTC/EFV (86\% versus $81 \%$ ) at week 48 for HIV RNA $<50$ copies/mL (difference $4.0 \%$, 95\% CI $-1.2 \%-9.2 \%$ ) per FDA snapshot analysis. Furthermore, superiority in efficacy was demonstrated for baseline HIV-1 RNA $\leq 100,000$ copies/mL $(n=508), 88 \%$ FTC/RPV/ TDF versus $81 \%$ EFV/FTC/TDF (difference $7.2 \%, 95 \% \mathrm{CI}$ $0.9 \%-13.4 \%$ ), and non-inferiority for $>100,000$ copies $/ \mathrm{mL}$ $(\mathrm{n}=276), 80 \% \mathrm{FTC} / \mathrm{RPV} / \mathrm{TDF}$ versus $82 \% \mathrm{EFV} / \mathrm{FTC} / \mathrm{TDF}$ (difference-1.8\%, 95\% CI-11.2\%-7.5\%). Overall, virologic failure, defined as HIV RNA $\geq 50$ copies/mL at week 48 , discontinuation due to lack of efficacy per investigator or discontinuation of study drug for reasons other than an adverse event with HIV RNA $\geq 50$ copies/mL was $8 \%$ for FTC/RPV/ TDF versus $6 \%$ for EFV/FTC/TDF (difference $2.7 \%, 95 \% \mathrm{CI}$ $-0.9 \%-6.3 \%)$. There were fewer study drug discontinuations due to adverse events in FTC/RPV/TDF than in EFV/FTC/ TDF. The STR FTC/RPV/TDF showed overall non-inferior efficacy and improved tolerability compared with the STR EFV/FTC/TDF, as well as superior efficacy for subjects with a baseline viral load $\leq 100,000$ copies/mL in treatment-naïve HIV-1-infected subjects. ${ }^{43}$

\section{Practical issue: selecting an NNRTI as the third agent in antiretroviral regimens for treatment-naïve patients}

Most guidelines recommend NNRTIs, PIs, or integrase inhibitors for use as the third agent in antiretroviral regimens., ${ }^{9,10}$ When comparing first- and second-generation NNRTIs, efavirenz is the preferred agent. However, when selecting an NNRTI as part of a treatment regimen, the characteristics of each individual antiretroviral agent should be considered, to tailor the appropriate treatment for a patient. Efavirenz has more CNS adverse events than others, and some patients may discontinue this agent due to this unfavorable effect; efavirenz should be avoided in patients with pre-existing psychiatric conditions. Furthermore, efavirenz has considerable adverse effects on lipid metabolism and leads to cutaneous eruptions. Nevirapine is an alternative to efavirenz in patients who experience adverse effects. Nevirapine also has some limitations, particularly severe hepatitis in patients with high CD4 levels ( $>250$ cells/ $\mathrm{mm}^{3}$ in females and $>400$ cells $/ \mathrm{mm}^{3}$ in males), and current guidelines recommend initiating ART at higher CD4 cell count levels than in the past. ${ }^{9}$ This guideline change may limit the use of nevirapine in patients unless they present with advanced disease. Nevirapine has less effect on lipid parameters than efavirenz, and no CNS adverse effects. Likewise, rilpivirine has a favorable effect on lipid profiles and also has no significant CNS side effects. However, it is clear that in subjects with a previous suboptimal response, and those with baseline viral loads $>100,000$ copies $/ \mathrm{mL}$, rilpivirine should be avoided. Other considerations include the requirement of food for absorption, and the contraindication in patients who are on rifamycins, such as rifampicin, rifabutin, or rifapentine, due to a significant drug interaction. ${ }^{44}$ When selecting an NNRTI for treatment-naïve 


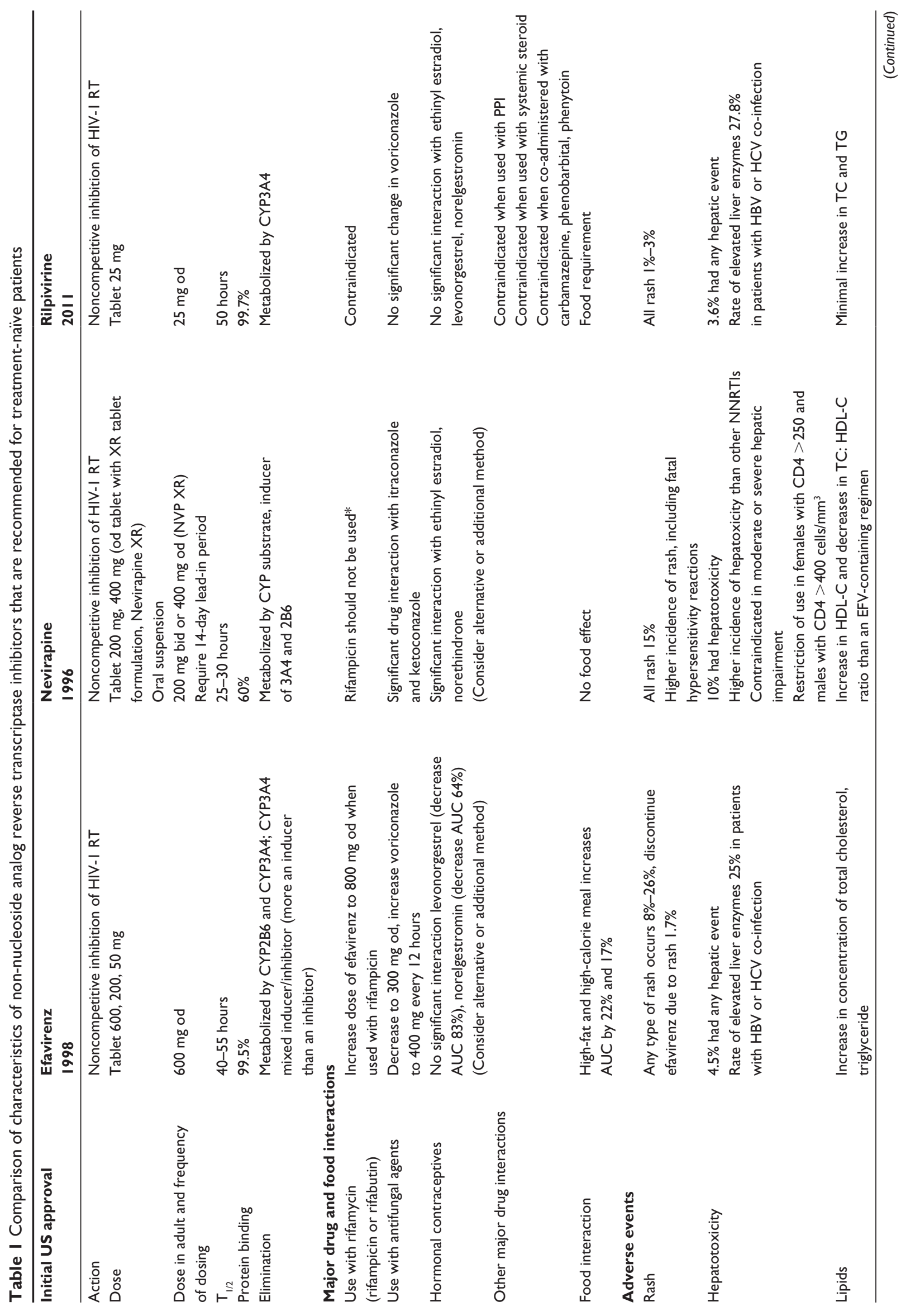




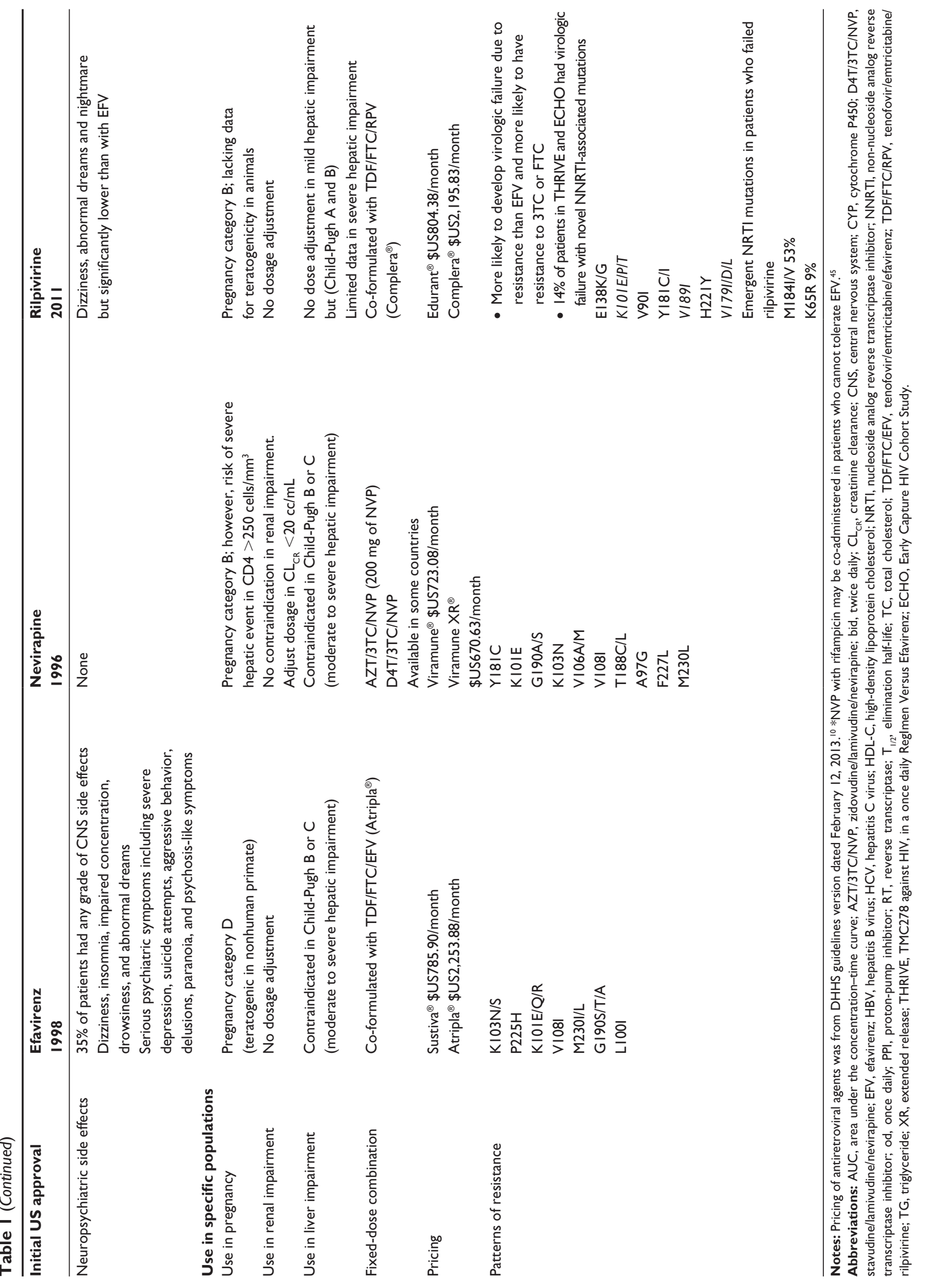


Table 2 Summary characteristics of rilpivirine $\left(\right.$ Edurant $\left.^{\circledR}\right)$

\begin{tabular}{|c|c|}
\hline Class & NNRTI \\
\hline Action & $\begin{array}{l}\text { Rilpivirine acts at hydrophobic position near NNRTI-binding site and causes inactivation of reverse } \\
\text { transcriptase enzyme }\end{array}$ \\
\hline Dose & 25 mg once daily, with food \\
\hline Formulation & Tablet, fixed-dose combination with TDF/FTC \\
\hline Time to maximal plasma concentration & $4-5$ hours \\
\hline Elimination half-life & Approximately 50 hours \\
\hline Dose in hepatic impairment & $\begin{array}{l}\text { - No dose adjustment in mild and moderate hepatic impairment (Child-Pugh class A and B) } \\
\text { - No clinical information in Child-Pugh class A }\end{array}$ \\
\hline Dose in renal impairment & $\begin{array}{l}\text { - No dose adjustment is required in mild to moderate renal impairment } \\
\text { - Require monitoring in severe or end-stage renal disease } \\
\text { - Rilpivirine is highly protein-bound and may not be significantly removed by hemodialysis or } \\
\text { peritoneal dialysis } \\
\text { - Higher risk of hepatitis in patients co-infected with HBV or HCV }\end{array}$ \\
\hline Use in pregnancy & Pregnancy category B \\
\hline Use in patients with tuberculosis & Contraindicated if co-administered with rifampicin and rifabutin, rifapentine \\
\hline Adverse effects & $\begin{array}{l}\text { Rash, depression, insomnia, headache } \\
\text { Use with caution when co-administered with drugs that prolong QTc }\end{array}$ \\
\hline Major drug interactions & $\begin{array}{l}\text { Acid-lowering agents such as antacid and H-receptor antagonists. RPV is contraindicated when } \\
\text { co-administered with PPI } \\
\text { Contraindicated when co-administered with } \\
\text { - Anticonvulsants: carbamazepine, oxcarbamazepine, Phenobarbital, and phenytoin } \\
\text { - Glucocorticoid (>one dose) systemic dexamethasone } \\
\text { - St John's wort } \\
\text { - Rifabutin }\end{array}$ \\
\hline $\begin{array}{l}\text { Conditions in which it should be } \\
\text { used with caution }\end{array}$ & Patients with baseline HIV-I viral load $>100,000$ copies $/ \mathrm{mL}$ due to possible suboptimal response \\
\hline Resistance pattern & 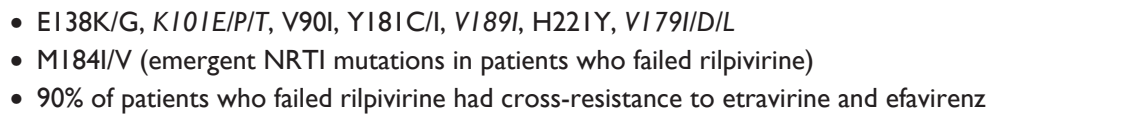 \\
\hline Price in the USA & $\begin{array}{l}\text { Edurant }^{\circledR} \text { (rilpivirine) } 30 \text { tabs } \$ 804.38 \\
\text { Complera }^{\circledR} \text { (TDF/FTC/RPV) } 30 \text { tabs } \$ 2,195.83\end{array}$ \\
\hline
\end{tabular}

Note: Data from. ${ }^{10}$

Abbreviations: $\mathrm{HBV}$, hepatitis B virus; $\mathrm{HCV}$, hepatitis C virus; NNRTI, non-nucleoside analog reverse transcriptase inhibitor; NRTI, nucleoside analog reverse transcriptase inhibitor; PPI, proton-pump inhibitor; QTc, corrected QT interval; TDF/FTC/RPV, tenofovir/emtricitabine/rilpivirine.

patients, the physician should compare the advantages and disadvantages of each individual agent. Table 1 shows a comparison of characteristics of NNRTIs currently recommended for treatment-naïve patients, and Table 2 shows the clinical characteristics of rilpivirine.

\section{Disclosure}

Kiat Ruxrungtham has received support from the Thailand Research Fund (TRF) Senior Researcher Scholar, and from the National Research University (NRU) Program of the CHE and the Ratchadaphiseksomphot Endowment Fund, Thailand, for grant HR1161A. He has also received speaker honoraria or educational or research grant support from Janssen-Cilag and Tibotec. Opass Putcharoen has received support by the Senior Researcher Scholar from the Thailand Research Fund (TRF) under KR's award. The authors report no other conflicts of interest in this work.

\section{References}

1. Chun TW, Carruth L, Finzi D, et al. Quantification of latent tissue reservoirs and total body viral load in HIV-1 infection. Nature. 1997;387:183-188.

2. Richman DD, Margolis DM, Delaney M, Greene WC, Hazuda D, Pomerantz RJ. The challenge of finding a cure for HIV infection. Science. 2009;323:1304-1307.

3. Pomerantz RJ, Horn DL. Twenty years of therapy for HIV-1 infection. Nat Med. 2003;9:867-873.

4. Young J, Rickenbach M, Weber R, et al. Body fat changes among antiretroviral-naïve patients on PI- and NNRTI-based HAART in the Swiss HIV cohort study. Antivir Ther. 2005;10:73-81.

5. Young J, Weber R, Rickenbach M, et al. Lipid profiles for antiretroviralnaïve patients starting PI- and NNRTI-based therapy in the Swiss HIV cohort study. Antivir Ther. 2005;10:585-591.

6. Gardner EM, Burman WJ, Maravi ME, Davidson AJ. Durability of adherence to antiretroviral therapy on initial and subsequent regimens. AIDS Patient Care STDS. 2006;20:628-636.

7. Chesney MA. Factors affecting adherence to antiretroviral therapy. Clin Infect Dis. 2000;30 Suppl 2:S171-S176.

8. Stone VE, Hogan JW, Schuman P, et al. Antiretroviral regimen complexity, self-reported adherence, and HIV patients' understanding of their regimens: survey of women in the her study. $J$ Acquir Immune Defic Syndr. 2001;28:124-131. 
9. Thompson MA, Aberg JA, Hoy JF, et al. Antiretroviral treatment of adult HIV infection: 2012 recommendations of the International Antiviral Society-USA panel. JAMA. 2012;308:387-402.

10. Panel on Antiretroviral Guidelines for Adults and Adolescents. Guidelines for the Use of Antiretroviral Agents in HIV-1-Infected Adults and Adolescents. Department of Health and Human Services; Rockville, MD. Available from: http://aidsinfo.nih.gov/contentfiles/lvguidelines/ adultandadolescentgl.pdf. Accessed March 1, 2013.

11. van Leth F, Phanuphak P, Ruxrungtham K, et al. Comparison of first-line antiretroviral therapy with regimens including nevirapine, efavirenz, or both drugs, plus stavudine and lamivudine: a randomised open-label trial, the 2NN Study. Lancet. 2004;363: $1253-1263$.

12. Clifford DB, Evans S, Yang Y, et al. Impact of efavirenz on neuropsychological performance and symptoms in HIV-infected individuals. Ann Intern Med. 2005;143:714-721.

13. Fundaro C, Genovese O, Rendeli C, Tamburrini E, Salvaggio E. Myelomeningocele in a child with intrauterine exposure to efavirenz. AIDS. 2002;16:299-300.

14. Molina JM, Andrade-Villanueva J, Echevarria J, et al. Once-daily atazanavir/ritonavir compared with twice-daily lopinavir/ritonavir, each in combination with tenofovir and emtricitabine, for management of antiretroviral-naïve HIV-1-infected patients: 96-week efficacy and safety results of the CASTLE study. J Acquir Immune Defic Syndr. 2010;53:323-332.

15. Mills AM, Nelson M, Jayaweera D, et al. Once-daily darunavir/ritonavir vs lopinavir/ritonavir in treatment-naïve, HIV-1-infected patients: 96-week analysis. AIDS. 2009;23:1679-1688.

16. Eron JJ Jr, Rockstroh JK, Reynes J, et al. Raltegravir once daily or twice daily in previously untreated patients with HIV-1: a randomised, active-controlled, phase 3 non-inferiority trial. Lancet Infect Dis. 2011;11:907-915.

17. Madruga JV, Cahn P, Grinsztejn B, et al. Efficacy and safety of TMC125 (etravirine) in treatment-experienced HIV-1-infected patients in DUET1:24-week results from a randomised, double-blind, placebo-controlled trial. Lancet. 2007;370:29-38.

18. Katlama C, Clotet B, Mills A, et al. Efficacy and safety of etravirine at week 96 in treatment-experienced HIV type-1-infected patients in the DUET-1 and DUET-2 trials. Antivir Ther. 2010;15:1045-1052.

19. Girard PM, Campbell T, Grinsztejn B, et al. Pooled week 96 results of the phase III DUET-1 and DUET-2 trials of etravirine: further analysis of adverse events and laboratory abnormalities of special interest. $H I V$ Med. 2012;13(7):427-435.

20. Janssen PA, Lewi PJ, Arnold E, et al. In search of a novel anti-HIV drug: multidisciplinary coordination in the discovery of 4-[[4-[[4-[(1E)-2cyanoethenyl]-2,6-dimethylphenyl]amino]-2- pyrimidinyl]amino]benzonitrile (R278474, rilpivirine). J Med Chem. 2005;48:1901-1909.

21. Das K, Bauman JD, Clark AD Jr, et al. High-resolution structures of HIV-1 reverse transcriptase/TMC278 complexes: strategic flexibility explains potency against resistance mutations. Proc Natl Acad Sci USA. 2008; $105: 1466-1471$.

22. Lansdon EB, Brendza KM, Hung M, et al. Crystal structures of HIV-1 reverse transcriptase with etravirine (TMC125) and rilpivirine (TMC278): implications for drug design. J Med Chem. 2010;53:4295-4299.

23. Goebel F, Yakovlev A, Pozniak AL, et al. Short-term antiviral activity of TMC278-a novel NNRTI-in treatment-naïve HIV-1-infected subjects. AIDS. 2006;20:1721-1726.

24. Wilkin A, Pozniak AL, Morales-Ramirez J, et al. Long-term efficacy, safety, and tolerability of rilpivirine (RPV, TMC278) in HIV type 1-infected antiretroviral-naïve patients: week 192 results from a phase IIb randomized trial. AIDS Res Hum Retroviruses. 2012;28:437-446.

25. Pozniak AL, Morales-Ramirez J, Katabira E, et al. Efficacy and safety of TMC278 in antiretroviral-naïve HIV-1 patients: week 96 results of a phase IIb randomized trial. AIDS. 2010;24:55-65.
26. Molina JM, Cahn P, Grinsztejn B, et al. Rilpivirine versus efavirenz with tenofovir and emtricitabine in treatment-naïve adults infected with HIV-1 (ECHO): a phase 3 randomised double-blind active-controlled trial. Lancet. 2011;378:238-246.

27. Cohen CJ, Andrade-Villanueva J, Clotet B, et al. Rilpivirine versus efavirenz with two background nucleoside or nucleotide reverse transcriptase inhibitors in treatment-naïve adults infected with HIV-1 (THRIVE): a phase 3, randomised, non-inferiority trial. Lancet. 2011;378:229-237.

28. Cohen CJ, Molina JM, Cahn P, et al. Efficacy and safety of rilpivirine (TMC278) versus efavirenz at 48 weeks in treatment-naïve HIV-1infected patients: pooled results from the phase 3 double-blind randomized ECHO and THRIVE Trials. J Acquir Immune Defic Syndr. 2012;60:33-42.

29. Cohen CJ, Molina JM, Cassetti I, et al. Week 96 efficacy and safety of rilpivirine in treatment-naïve, HIV-1 patients in two Phase III randomised trials. AIDS. 2013;27(6):939-950.

30. Hu Z, Kuritzkes DR. Interaction of reverse transcriptase (RT) mutations conferring resistance to lamivudine and etravirine: effects on fitness and RT activity of human immunodeficiency virus type 1. J Virol. 2011;85:11309-11314

31. Tambuyzer L, Vingerhoets J, Azijn H, et al. Characterization of genotypic and phenotypic changes in HIV-1-infected patients with virologic failure on an etravirine-containing regimen in the DUET-1 and DUET-2 clinical studies. AIDS Res Hum Retroviruses. 2010;26:1197-1205.

32. Vingerhoets J, Azijn H, Tambuyzer L, et al. Short communication: activity of etravirine on different HIV type 1 subtypes: in vitro susceptibility in treatment-naïve patients and week 48 pooled DUET study data. AIDS Res Hum Retroviruses. 2010;26:621-624.

33. Wheeler WH, Ziebell RA, Zabina H, et al. Prevalence of transmitted drug resistance associated mutations and HIV-1 subtypes in new HIV-1 diagnoses, US-2006. AIDS. 2010;24:1203-1212.

34. Wittkop L, Günthard HF, de Wolf F, et al. Effect of transmitted drug resistance on virological and immunological response to initial combination antiretroviral therapy for HIV (EuroCoord-CHAIN joint project): a European multicohort study. Lancet Infect Dis. 2011;11:363-371.

35. Vingerhoets J, Rimsky L, Van Eygen V, et al. Preexisting mutations in the rilpivirine Phase III trials ECHO and THRIVE: prevalence and impact on virologic response. Antivir Ther. 2013;18(2):253-256.

36. Hodder S, Arasteh K, De Wet J, et al. Effect of gender and race on the week 48 findings in treatment-naïve, HIV-1-infected patients enrolled in the randomized, phase III trials ECHO and THRIVE. HIV Med. 2012;13:406-415.

37. Nelson M, Amaya G, Clumeck N, et al. Efficacy and safety of rilpivirine in treatment-naïve, HIV-1-infected patients with hepatitis B virus/ hepatitis $\mathrm{C}$ virus coinfection enrolled in the Phase III randomized, double-blind ECHO and THRIVE trials. J Antimicrob Chemother. 2012;67:2020-2028.

38. Taylor GP, Clayden P, Dhar J, et al. British HIV Association guidelines for the management of HIV infection in pregnant women 2012. HIV Med. 2012;13 Suppl 2:87-157.

39. World Health Organization. Antiretroviral Drugs for Treating Pregnant Women and Preventing HIV Infection in Infants: Guidelines on Care, Treatment and Support for Women Living with HIV/AIDS and their Children in Resource-Constrained Settings. Geneva: World Health Organization; 2004. Available from: http://www.who.int/hiv/pub/mtct/ guidelines/en/. Accessed March 22, 2013.

40. Palella F, Tebas P, Gazzard B, et al. SPIRIT study: switching to emtricitabine/rilpivirine/tenofovir df (FTC/RPV/TDF) single-tablet regimen (STR) from a ritonavir-boosted protease inhibitor and two nucleoside reverse transcriptase inhibitors (NRTIS) maintains HIV suppression and improves serum lipids. 19th International AIDS Conference. July 22-27, 2012; Washington DC. Abstract TUAB0104. 
41. Mills A, Cohen C, DeJesus E, et al. Switching from efavirenz/ emtricitabine/tenofovir disoproxil fumarate (EFV/FTC/TDF) single tablet regimen (STR) to emtricitabine/rilpivirine/tenofovir disoproxil fumarate (FTC/RPV/TDF) STR in virologically suppressed, HIV-1 infected subjects. 51st Interscience Conference on Antimicrobial Agents and Chemotherapy (ICAAC); September 17-20, 2011; Chicago, IL.

42. Rimsky L, Vingerhoets J, Van Eygen V, et al. Genotypic and phenotypic characterization of HIV-1 isolates obtained from patients on rilpivirine therapy experiencing virologic failure in the phase $3 \mathrm{ECHO}$ and THRIVE studies: 48-week analysis. J Acquir Immune Defic Syndr. 2012;59:39-46.

43. Cohen C, Wohl D, Arribas J, et al. STAR Study: single tablet regimen emtricitabine/rilpivirine/tenofovir DF is non-inferior to efavirenz/ emtricitabine/tenofovir DF in ART-naïve adults. $J$ Int AIDS Soc. 2012;15 Suppl 4:18221.
44. Crauwels H, van Heeswijk R, Kestens D, et al. The pharmacokinetic (PK) interaction between rifabutin and TMC278, an investigational nonnucleoside reverse transcriptase inhibitor (NNRTI). AIDS 2008 - XVII International AIDS Conference; August 3-8, 2008; Mexico City, MX. Abstract TUPE0080.

45. Manosuthi W, Tantanathip P, Chimsuntorn S, et al. Treatment outcomes of patients co-infected with HIV and tuberculosis who received a nevirapine-based antiretroviral regimen: a four-year prospective study. Int J Infect Dis. 2010;14:e1013-e1017.
HIV/AIDS - Research and Palliative Care

\section{Publish your work in this journal}

HIV/AIDS - Research and Palliative Care is an international, peerreviewed open-access journal focusing on advances in research in HIV, its clinical progression and management options including antivira treatment, palliative care and public healthcare policies to contro viral spread. The journal welcomes original research, basic science,
Dovepress

clinical \& epidemiological studies, reviews \& evaluations, expert opinion \& commentary, case reports \& extended reports. The manuscript management system is completely online and includes a very quick and fair peer-review system. Visit http://www.dovepress.com/ testimonials.php to read real quotes from published authors.

Submit your manuscript here: http://www.dovepress.com/hivaids---research-and-palliative-care-journal 